\title{
IMPLEMENTAÇÃO BIM NA NRV | NORVIA
}

\author{
Luís Pedro Bidarra $^{(1)}$, Ana Clemente ${ }^{(1)}$, António Costa ${ }^{(1)}$ \\ (1) NRV - Consultores de Engenharia, SA
}

\begin{abstract}
Resumo
A implementação BIM na NRV tem como objetivo dotar a empresa de meios e processos conducentes à conceção de projetos segundo a metodologia de trabalho Building Information Modeling, tendo como premissas a imagem e a qualidade dos projetos produzidos com a atual metodologia e tendo em vista um aumento de eficiência na sua elaboração, assim como o aumento da competitividade da empresa num mercado cada vez mais tecnológico.

O processo de implementação de tecnologias BIM numa empresa de projeto é por si só árduo, ganha contornos ainda mais complexos numa empresa multidisciplinar como a NRV, com diversas áreas de atuação.

Todo este processo obrigou a uma profunda reformulação na forma como abordamos os projetos, desde a alteração dos campos de ação dos intervenientes, passando pela aquisição de meios, conceção e documentação de processos internos, formação e qualificação dos recursos e até o desenvolvimento de software específico.

$\mathrm{O}$ presente artigo pretende descrever a abordagem da NRV à implementação BIM, dando especial ênfase à evolução processual observada nos projetos BIM entretanto desenvolvidos. Salientam-se os principais resultados operacionais alcançados, dificuldades e benefícios encontrados, assim como a avaliação dos ganhos de eficiência efetivos e os desenvolvimentos futuros por forma a automatizar e assim tornar o processo BIM na NRV cada vez mais eficiente.
\end{abstract}

\section{Enquadramento da mudança para BIM}

A NRV é uma consultora de engenharia multidisciplinar com mais de 30 anos que desenvolve a sua atividade em vários domínios: vias de comunicação, infraestruturas urbanas e saneamento básico, aeroportos, obras marítimas e portuárias, edifícios e instalações industriais, ordenamento do território, estruturas especiais (barragens, pontes e túneis), topografia e geotecnia.

A vasta experiência nacional e internacional da NRV permitiu-lhe reconhecer a emergente importância da implementação da metodologia de trabalho Building Information Modeling como garante da manutenção da empresa na vanguarda das mais recentes tendências 
tecnológicas da indústria AEC (Arquitetura, Engenharia e Construção), particularmente em mercados mais desenvolvidos como o europeu, norte-americano ou o médio-oriente.

Embora os mercados supramencionados não representem um grande volume de negócios para a empresa neste momento, e de em muitos dos mercados onde a empresa atua o BIM não se apresentar como uma necessidade imediata, a mudança do processo produtivo da empresa para a metodologia de trabalho BIM tem em vista a otimização deste, com ganhos de eficiência efetivos, nomeadamente: o aumento do nível de conformidade entre documentos que têm como origem o modelo BIM, a deteção de problemas e incompatibilidades mais cedo durante o projeto, a coordenação entre especialidades através do modelo BIM e a comunicação com o cliente.

A aposta na inovação, área onde se insere o universo BIM, é encarada como uma necessidade e oportunidade da empresa a participar em projetos aliciantes em mercados exigentes, refletindo-se internamente num processo produtivo muito mais eficiente, numa melhor coordenação entre especialidades e numa melhor gestão da informação digital produzida [1]. Assim, este artigo tem em vista descrever o processo de implementação assim como a estratégia da NRV para operacionalizar a transição do processo tradicional CAD para a metodologia de trabalho BIM.

\section{Implementação e estratégia BIM}

A implementação BIM no seio de uma empresa multidisciplinar com a dimensão da NRV reveste-se de complexidades de várias ordens e implica sempre um investimento em aquisição de software, melhoria de hardware, formação, contratação de pessoal e quebras de produtividade iniciais [2]. Assim, é imperativa uma gestão estratégica dos investimentos por forma a que a transição e a manutenção da metodologia de trabalho seja sustentável a longo prazo [3].

Uma das etapas inerentes à implementação da tecnologia BIM é sem dúvida a escolha do software a utilizar. No caso da NRV, as escolhas recaíram sobre o Autodesk Civil 3D, para projetos de infraestruturas e vias de comunicação e no Autodesk Revit para projetos de estruturas e especialidades em edifícios. A escolha do Autodesk Civil 3D foi alicerçada no facto de, para além de já haver algumas licenças adquiridas, existir algum know-how adquirido na realização de projetos de drenagem de águas residuais e de vias de comunicação, que já haviam sido desenvolvidos nesta plataforma. No caso do Autodesk Revit a escolha prendeu-se essencialmente na versatilidade e a multidisciplinaridade do software, nomeadamente na modelação de MEP (Mechanical, Electical and Plumbing), conseguindo-se assim, com um mesmo software, modelar todas as especialidades de projeto de edifícios que a NRV desenvolve. Foram também fatores importantes para decisão da escolha do Autodesk Revit a adaptação da empresa as ferramentas Autodesk, assim como a constatação da quota de mercado que o Autodesk Revit possui não só no mercado nacional como em mercados internacionais. A aquisição de licenças dos softwares BIM é um processo oneroso, pelo que é necessário um trabalho de racionalização de recursos que vá de encontro às necessidades de modelação de cada divisão da empresa e sobretudo, que seja financeiramente sustentável. Assim, as especialidades MEP trabalham com a versão do Revit completa, já que apenas esta versão permite e modelação deste tipo de sistemas. Por sua vez, a divisão de estruturas utiliza licenças Revit LT em fases iniciais dos projetos, transitando para a versão completa nas fases finais e 
em projetos de maiores dimensões. Esta opção permitiu poupanças significativas nos custos de licenciamento sem grandes alterações produtivas, sendo que as funcionalidades da versão LT são suficientes para todo o processo de modelação estrutural se não forem incluídas armaduras. Foi adiada a implementação da modelação de armaduras por se considerar, a este ponto, ineficiente face as ferramentas já desenvolvidas em AutoCAD para o efeito.

Uma problemática central em qualquer processo de implementação é a metodologia de distribuição de recursos humanos. O ponto de partida para o processo de implementação BIM passou pela contratação de um BIM Manager, com formação especializada em BIM, para conduzir e gerir o processo de implementação BIM da empresa.

Após a elaboração do plano de implementação, foram criados dois grupos de trabalho multidisciplinares (um relativo ao projeto de vias de comunicação e infraestruturas e outro relativo ao projeto de edifícios) com atuação na criação de conteúdos e procedimentos BIM, com coordenação através de reuniões periódicas, lideradas pelo BIM Manager.

O desenvolvimento de documentos normativos, standards e boas práticas de apoio à modelação e gestão dos respetivos fluxos de trabalho, é decisivo para uma implementação bem-sucedida dos processos BIM. Todos os documentos normativos e fluxos de trabalho foram desenvolvidos de acordo com as normas BIM britânicas [4], pois serão as mais próximas das futuras normas ISO internacionais relativas ao método BIM.

Assim e com o objetivo de estruturar o processo de implementação e facilitar a utilização das ferramentas BIM foram desenvolvidos um conjunto alargado de documentos estruturantes e ferramentas facilitadoras dos processos BIM, designadamente:

- $\quad$ Plano de Implementação BIM: documento que visa identificar os objetivos a curto, médio e longo prazo da implementação BIM, assim como o seu valor estratégico. Detalha também todos os processos e infraestrutura conducentes à operacionalização da implementação BIM;

- Guia de Contratação BIM: documento de apoio à formalização de propostas em BIM;

- $\quad$ Plano de Execução BIM: documento onde são definidos, para cada projeto BIM, os requisitos do modelo, as regras de modelação a respeitar, o nível de desenvolvimento necessário para cada elemento em cada fase do projeto, o conteúdo a modelar por cada especialidade, a calendarização das atividades assim como as responsabilidades dos vários intervenientes;

- Manuais de boas práticas de modelação e trocas de informação em BIM: documento onde são fixadas um conjunto de regras mínimas que contribuam para que os modelos BIM sejam desenvolvidos de forma eficiente e estejam de acordo com os formatos requeridos;

- Manuais de interoperabilidade entre softwares BIM e softwares de cálculo: documentos que visam definir regras de modelação e exportação para assegurar uma correta transmissão da informação entre os modelos BIM e os softwares de cálculo.

Foram também criados um conjunto de elementos-chave, utilizados diretamente pelas ferramentas de modelação e aplicações BIM e que servem de suporte ao desenvolvimento dos modelos BIM das várias especialidades, destacando-se os seguintes: 
- $\quad$ Templates: os templates foram criados tendo como premissa a imagem de projeto das várias divisões da NRV, por forma a agilizar a introdução de dados e extração de peças desenhadas nos softwares BIM;

- Detalhes-Tipo: detalhes 2D paramétricos que podem ser utilizados em vários projetos diferentes agilizando o tempo de produção das peças desenhadas;

- $\quad$ Biblioteca de Objetos BIM: foram desenvolvidos objetos paramétricos BIM que vão de encontro aos requisitos de imagem de projeto da NRV, assim como aos requisitos de desenvolvimento definidos nos planos de implementação e execução BIM.

Face à diversidade de objetos desenvolvidos, resultantes da multidisciplinaridade da NRV, e por forma a fomentar a internacionalização da NRV através da tecnologia BIM, achou-se relevante atuar sobre a Biblioteca de Objetos BIM a dois níveis: na classificação de objetos segundo normas internacionais e na nomenclatura destes.

Deste modo, foi efetuado um estudo comparativo dos sistemas de classificação internacionais de objetos BIM por forma a garantir que o sistema de classificação escolhido vá de encontro as necessidades e exigências dos mercados internacionais. Concluiu-se então que o sistema mais adequado a utilizar seria a Uniclass [5] pois, para além das suas características, onde se destaca a sua leitura e aplicabilidade em objetos, está em linha com a opção da adoção de normalização britânica que num futuro próximo se pensa estar mais próxima da normalização ISO internacional [6].

No que concerne a nomenclatura de objetos BIM, era também imperativo atuar sobre uma codificação de nomenclatura que fosse transversal a toda a empresa e que simultaneamente fosse de encontro à normalização britânica adotada na empresa. A escolha recaiu assim na proposta de nomenclatura da BS 8541-1 [7], por se considerar ser a mais abrangente e de fácil leitura por todos os intervenientes no processo BIM.

É ainda importante ressalvar que no seio da empresa o processo de criação de famílias é totalmente livre e encorajado a todos os intervenientes no processo BIM, sendo a sua aprovação e colocação na Biblioteca de Objetos BIM restrita ao BIM Manager.

Sendo os objetos BIM e a sua correta utilização o garante da qualidade do modelo BIM na sua globalidade é imperativo o controlo da qualidade de todos objetos utilizados no processo BIM. Este papel fulcral que os objetos BIM representam no processo BIM motivou o desenvolvimento de uma aplicação informática que garanta a utilização exclusiva dos objetos BIM constantes na Biblioteca de Objetos da empresa, previamente validados pelo BIM Manager.

\section{Formação e trocas de informação em BIM}

O carácter disruptivo da alteração de metodologia de trabalho para a metodologia de trabalho BIM compreende uma série de desafios dos quais se destacam particularmente os desafios relacionados com a componente humana, onde se incluem aspetos sobre formação, facilidade de aquisição de competências, perfil tecnológico das pessoas, motivação e incentivos à adoção, assim como os desafios relacionados com a metodologia e a sistematização de processos, sobretudo no que diz respeito a questões de interoperabilidade, comunicação e troca de dados [8]. 
A integração de processos BIM depende, portanto, da capacidade das equipas em adotar novos processos de trabalho, isto é, absorver a nova forma de trabalhar e aplicá-la no desenvolvimento das suas atividades. Trata-se de um aspeto muito importante na implementação de processos BIM que tende a ser bastante condicionada pelo calendário de trabalho das equipas, que têm de conciliar atividades formativas com atividades de produção. A vertente humana e processual desempenha assim um papel fulcral no processo de implementação BIM.

Foram definidas equipas multidisciplinares, constituídas por engenheiros e desenhadores, em cada divisão, com base no seu perfil tecnológico e foram desenvolvidos programas de formação modulares, adaptados às necessidades e aos fluxos de trabalho em BIM para cada divisão, que abordaram não só aspetos de ordem mais prática, de manuseamento dos softwares BIM, mas também aspetos de ordem mais teórica e mais global acerca da metodologia de trabalho BIM. As formações foram ministradas pelo BIM Manager pois, para além de estar por trás de todo o desenvolvimento de material, documentação e fluxos de trabalho em BIM, já tinha experiência como formador na área do BIM.

Numa consultora de engenharia como a NRV, onde o principal objetivo é o desenvolvimento de projetos em BIM, o foco do processo de implementação deve estar na criação de competências de modelação. Assim, é fundamental que as equipas desenvolvam autonomia para utilizar o modelo BIM de modo a conseguir representar as soluções concebidas. Esta autonomia deve traduzir-se na capacidade de produzir modelos consistentes e robustos, de extrair desenhos, informação e quantidades do modelo, e de identificar o nível de desenvolvimento necessário para o modelo em função das exigências do projeto.

É também essencial que todas as partes envolvidas dominem os conceitos teóricos inerentes ao método de trabalho BIM, particularmente no que concerne ao ecossistema colaborativo BIM de troca e partilha de informação. Assim, todos os projetos piloto desenvolvidos em contexto de formação foram já desenvolvidos segundo a lógica colaborativa preconizada na metodologia de trabalho BIM.

Achou-se também relevante que todas as pessoas envolvidas no processo produtivo possuíssem conhecimentos em coordenação de modelos BIM, não só com as especialidades realizadas internamente, como também com os modelos externos. Esta medida não visa remover a figura do coordenador de projeto do processo produtivo em BIM, visa essencialmente sensibilizar todos os intervenientes no processo BIM para a problemática da coordenação de projetos, que com a adoção da metodologia de trabalho BIM se pretende tornar mais eficiente, diminuindo o trabalho de coordenação outrora necessário na metodologia de trabalho tradicional e consequentemente minimizando o trabalho de correções/revisões de projeto.

\section{Investigação e Desenvolvimento em BIM}

A criação de um grupo de Investigação e Desenvolvimento, para além dos grupos de trabalho BIM, visa tornar o processo BIM cada vez mais eficiente no seio da empresa, através otimização e automatização de processos, seja por via da programação, seja por integração de novas aplicações informáticas ou metodologias nos métodos de trabalho internos. Este grupo é para já constituído pelo BIM Manager e por um engenheiro informático.

Foram desenvolvidos com sucesso duas aplicações informáticas para responder a duas necessidades que se acharam prementes no processo de implementação BIM e que não existiam no mercado com a configuração e nível de personalização que se pretendia. 
No decorrer do processo de implementação BIM nas especialidades MEP constatou-se que o mecanismo que o Revit possui para realizar o dimensionamento de tubagens conduz a valores substancialmente diferentes dos existentes nos regulamentos portugueses.

Numa perspetiva de ganhos de eficiência no processo produtivo, e tendo em conta que no processo de criação dos objetos BIM já se havia tido em conta os caudais e os diâmetros das tubagens, considerou-se importante o desenvolvimento de uma aplicação informática que simultaneamente efetuasse o dimensionamento hidráulico das tubagens, assim como realizasse a verificação da pressão hidráulica no ponto mais desfavorável da rede, para cumprir os critérios de conforto na rede no que concerne às pressões.
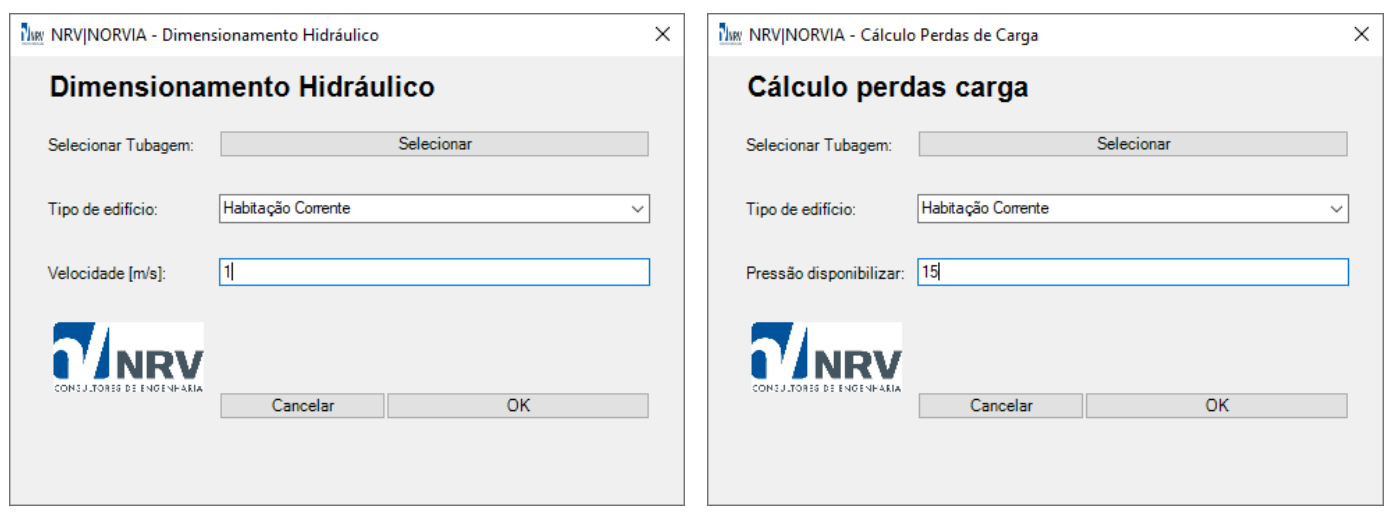

Figura 1: Aplicações informáticas para dimensionamento e verificação da pressão na rede.

Esta aplicação informática foi desenvolvido através interface de uma interface de programação gráfica do Revit, o Dynamo, que permite de uma forma mais fácil e mais ágil, o desenvolvimento de aplicações informáticas através de programação visual, sem a necessidade de programação textual. A ferramenta de programação visual Dynamo é distribuída com a versão completa do Revit.

Uma dificuldade recorrente na gestão diária de projetos BIM é a manutenção da qualidade dos objetos criados. Comportamentos como a cópia de outros projetos, alterações, utilização sem conhecimento prévio do modo de parametrização e utilização direta dos objetos BIM de fabricantes dificultavam a concretização do projeto BIM, reduzindo a qualidade do produto final.

A perceção da importância do controlo da qualidade associado à utilização dos objetos BIM, motivou o desenvolvimento de uma aplicação informática que centralizasse numa mesma plataforma, a biblioteca de objetos BIM já desenvolvida e validada pelo BIM Manager.

Esta aplicação informática foi desenvolvido recorrendo a API (Application Programming Interface) do Revit. Do ponto de vista informático a API corresponde, simplificadamente, a um conjunto de funções do software, acessíveis pelo utilizador através de programação, que permitem automatizar ou estender as funcionalidades nativas do Revit. Neste pressuposto e com recurso a programação na linguagem C\#, desenvolveu-se uma aplicação informática em duas versões, na forma de add-in para Revit, para utilização pelas versões completas de Revit e na versão aplicação independente para utilização pelas versões LT do Revit. O desenvolvimento da aplicação informática em duas versões distintas prende-se com o facto da versão LT do Revit não disponibilizar o acesso a API do Revit para o utilizador. 


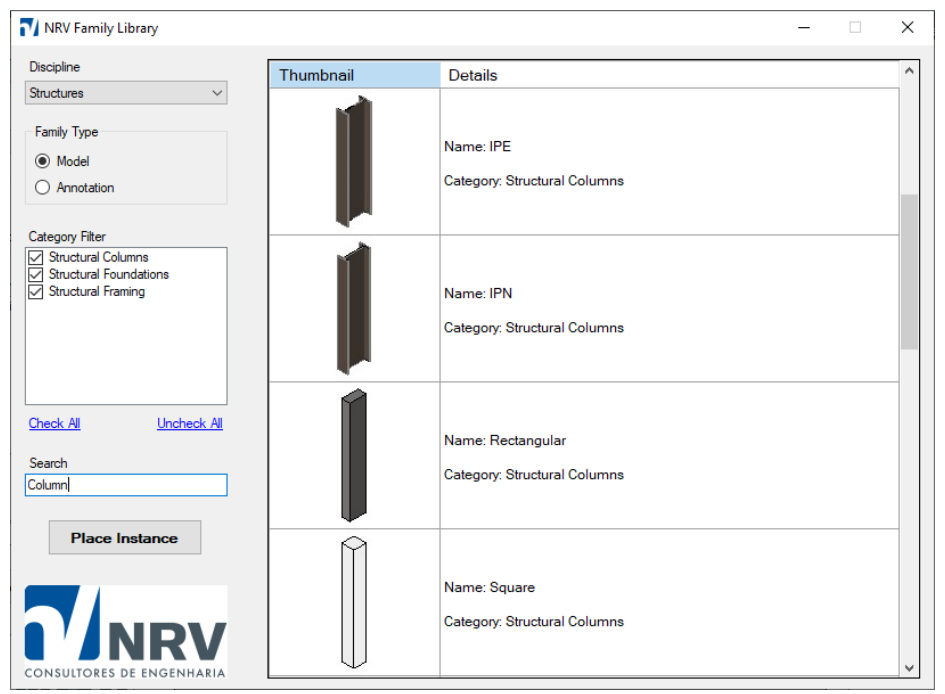

Figura 2: Biblioteca de objetos BIM.

O desenvolvimento de uma aplicação informática que pudesse simultaneamente operar nas versões completas e LT do Revit revestiu-se de complexidades, nomeadamente no que concerne à extração da informação de cada objeto BIM. Foi então necessário explorar não só a API do Revit, mas também a estrutura dos ficheiros de Revit por forma a dotar a aplicação informática de capacidade de interpretação da informação neles contidos sem necessitar de abrir os ficheiros.

A aplicação informática permite assim aos utilizadores a inserção dos objetos BIM no software de uma forma mais ágil e garantindo que toda a equipa está a utilizar a mesma biblioteca de objetos.

Pelas razões descritas e tendo em conta a falta de soluções no mercado que desempenhassem as funções deste conjunto de aplicações informáticas considera-se que o investimento no seu desenvolvimento foi bastante rentável.

\section{Desenvolvimentos futuros e compromisso BIM}

A implementação efetuada permite o desenvolvimento de projetos em tecnologia BIM em edifícios, em projetos viários, de infraestruturas urbanas e de saneamento básico. No entanto, existem áreas de projeto onde a possibilidade de otimização do processo de cálculo, desenho e comunicação é evidente.

Destaca-se particularmente a área do projeto de obras de arte, como pontes e túneis. Esta área foi identificada como um dos próximos desafios de implementação do método BIM. A aplicação do método BIM a este tipo de estruturas reveste-se de dificuldades, não só pela complexidade geométrica, mas também pelo nível de detalhe necessário, assim como pelo facto de serem estruturas muito menos tipificadas que as estruturas de edifícios [9].

No nosso entender, a chave para abordar a implementação BIM nas obras de arte está no designado design computacional [10]. O design computacional é definido como a implementação de estratégias computacionais para assistir o processo produtivo em BIM, o que a nosso ver pode ser operacionalizado por exemplo com a implementação da ferramenta de programação visual Dynamo, já utilizada anteriormente com sucesso no âmbito de 
desenvolvimento de aplicações informáticas e que neste âmbito pode ser utilizada como geradora das geometrias complexas e como automatização de processos em obras de arte de grandes dimensões.

Salienta-se também o potencial de desenvolvimento da área da topografia e cartografia onde a NRV já dispõe de equipamento de laser scanning e de recolha aérea de imagens por fotogrametria. A utilização destas tecnologias aliadas a metodologia de trabalho BIM pode ser amplamente otimizada através do desenvolvimento de software que, com recurso a inteligência artificial permita o automático reconhecimento não só dos elementos construtivos de um edifício, assim como das suas tubagens ou condutas, tornando assim mais eficiente a modelação do existente [11].

Outro dos desafios que se antevê no horizonte BIM da NRV é a aplicação dos conceitos de facility management aos estudos de conservação e reabilitação de pavimentos rodoviários [12]. Depois da implementação BIM nas obras viárias e da consequente produção de modelos BIM, o próximo passo será o desenvolvimento de fluxos de trabalho conducentes à gestão da fase de operação das estruturas viárias. Este conceito será também ser alargado aos restantes âmbitos de projeto da NRV.

Para além dos desafios supramencionados, muitos outros com certeza surgirão num futuro próximo. O BIM Manager, os grupos BIM e em particular o grupo de Investigação e Desenvolvimento, manterão o compromisso BIM no seio da NRV associado ao desenvolvimento de metodologias, processos e aplicações informáticas para que o processo produtivo em BIM seja o mais automatizado e eficiente possível.

\section{Conclusões}

A implementação BIM na NRV tem-se revelado um desafio interessante, possibilitando a interação profunda entre os vários âmbitos de projeto que a empresa desenvolve.

De entre as principais vantagens e benefícios da adoção do método BIM, destaca-se o aumento na eficiência dos processos internos. Foi este o principal foco do processo de implementação, pois nesta fase transitória que a indústria da construção atravessa, é a nosso ver o melhor caminho a seguir para ter o retorno do investimento da implementação do método BIM.

Ainda assim, destacam-se outros importantes benefícios diretos ou indiretos que se obtiveram com a implementação BIM:

- Adequação tecnológica do processo produtivo da empresa em linha com as tendências da indústria da AEC e preparando uma mudança de paradigma no setor da construção que se antevê inevitável;

- Aumento generalizado na qualidade e nível de detalhe dos projetos desenvolvidos, devido essencialmente a uma melhor coordenação de projetos e consequentemente a redução das incompatibilidades entre espacialidades;

- Melhoria da interoperabilidade entre os softwares de modelação e os softwares de cálculo;

- Aumento generalizado da precisão e eficiência na extração de quantidades;

- Agilização da capacidade de resposta a eventuais alterações ou revisões de projeto;

- Melhoria generalizada da capacidade de comunicação com todos os intervenientes no processo. 
Em suma, as orientações e objetivos inicialmente definidos permitiram que hoje seja possível identificar os índices de sucesso perspetivados.

Assim, conclui-se que a implementação BIM é definitivamente uma mais-valia para a empresa, possibilitando um aumento do número de clientes internos e a expansão para outros mercados externos.

Salienta-se que o BIM na NRV é um compromisso de futuro, automatizando e aumentando cada vez mais a eficiência do processo produtivo da empresa.

\section{Referências}

[1] P. Smith, "BIM implementation - Global strategies," Procedia Eng., vol. 85, pp. 482492, 2014, doi: 10.1016/j.proeng.2014.10.575.

[2] M. Baldwin, The BIM Manager: A Practical Guide for BIM Project Management, 1st ed. Berlin: Mensch und Maschine Scheiz AG, 2019.

[3] M. F. Antwi-Afari, H. Li, E. A. Pärn, and D. J. Edwards, "Critical success factors for implementing building information modelling (BIM): A longitudinal review," Autom. Constr., vol. 91, no. March, pp. 100-110, 2018, doi: 10.1016/j.autcon.2018.03.010.

[4] M. Richards, "Building information management: A standard framework and guide to BS 1192 BSI Standards," BSI Stand. Publ., no. 1, pp. 1-68, 2013.

[5] K. Afsari and C. M. Eastman, "A Comparison of Construction Classification Systems Used for Classifying Building Product Models,” 52nd ASC Annu. Int. Conf. Proc., no. 2001, pp. 1-8, 2016.

[6] Autodesk, "Classification Systems and Their Use in Autodesk Revit ${ }^{\circledR}$ Managing the 'I' in BIM," p. 42, 2017.

[7] BSI Committee B/555, "BS 8541-1:2012 Library objects for architecture, engineering and construction Part 1 : Identification and classification - Code of practice," Bs, vol. 8541-1, 2012.

[8] V. Singh, N. Gu, and X. Wang, "A theoretical framework of a BIM-based multidisciplinary collaboration platform," Autom. Constr., vol. 20, no. 2, pp. 134-144, 2011, doi: 10.1016/j.autcon.2010.09.011.

[9] C. S. Shim, N. R. Yun, and H. H. Song, "Application of 3D bridge information modeling to design and construction of bridges," Procedia Eng., vol. 14, pp. 95-99, 2011, doi: 10.1016/j.proeng.2011.07.010.

[10] M. Khaja, J. D. Seo, and J. J. McArthur, “Optimizing BIM Metadata Manipulation Using Parametric Tools," Procedia Eng., vol. 145, no. December, pp. 259-266, 2016, doi: 10.1016/j.proeng.2016.04.072.

[11] V. Ptrroucean, I. Armeni, M. Nahangi, J. Yeung, I. Brilakis, and C. Haas, "State of research in automatic as-built modelling," Adv. Eng. Informatics, vol. 29, no. 2, pp. 162171, 2015, doi: 10.1016/j.aei.2015.01.001.

[12]F. Tang, T. Ma, Y. Guan, and Z. Zhang, "Parametric modeling and structure verification of asphalt pavement based on BIM-ABAQUS," Autom. Constr., vol. 111, no. June 2019, p. 103066, 2020, doi: 10.1016/j.autcon.2019.103066. 